\title{
Some late-term thoughts of a density-functional theorist
}

\author{
ROBERT G PARR \\ Department of Chemistry, University of North Carolina, Chapel Hill, North Carolina 27599, USA \\ e-mail: rgparr@email.unc.edu
}

\begin{abstract}
Fourteen problems are stated within the density-functional theory of molecular electronic structure. Their alleviation will bring the subject closer to maturity.
\end{abstract}

Keywords. Density-functional theory; molecular electronic structure; quantum chemistry; molecular behavior.

\section{Preface}

Density-functional theory (DFT) of the electronic structure of molecules constitutes an important branch of quantum chemistry, now widely employed both for calculation on molecules and for discussion of molecular behavior.

Just a couple of decades ago, DFT was not providing a good tool for accurate chemical calculations. But this drastically changed. For instance, reacting to a certain lovely Journal of Chemical Physics paper about electron correlation, in 1988 at the University of North Carolina, we produced a good DFT way to compute correlation energy, which soon with an add-on from Canada grew into a scheme called BLYP. So far this paper has been cited more than twelve thousand times. Presently, computational DFT is thriving.

On the descriptive side, in "conceptual DFT", thousands of papers have been published, as well as special issues of journals, many reviews, and several books. Important ideas from the past have emerged in DFT, sifted, rehashed, and made more rigorous. New DFT concepts and principles also have arrived on the scene, importantly including new variational principles. The DFT of these things is strikingly like classical thermodynamics, where great progress came through careful attention to identifying the pertinent state variables in each particular circumstance of interest and deriving the consequences of the laws of thermodynamics in each case. Highly significant for the microscopic DFT we are discussing, we have, as we have had classically, the extraordinary concept of chemical potential. There is, as there was classically, focus on what might or might not actually happen in a given chemical situation, with the emphasis on what may or may not happen spontaneously.
Molecular DFT applies to a heterogeneous microscopic world more complicated than the macroscopic world of classical thermodynamics for homogeneous systems. Thermo presents at best an analogy for DFT, and so one expects to meet pitfalls and dead ends when one reaches for too much in developing DFT. The most appropriate density-functional language does not come easily. It is not just telling how to calculate better and to calculate more. For, as Ernest Davidson once said, "It is the words that are important, not the numbers".

A remarkable aspect of the present immature state of DFT, and a reason for optimism about the future of the subject, is that it already is producing fine numerical results and excellent qualitative descriptions of molecules. To work in such a great problem area is challenging and enjoyable.

\section{Fourteen problems in density-functional theory}

(1) Monotonicity: Prove that the ground-state electron density for any atom is monotonically decreasing away from the nucleus.

(2) Overlap integrals between atoms-in-molecules: In some definitions of atoms-in-molecules (AIM), overlaps between "atoms" are zero; in some they are not. Compute, compare, and discuss the overlap integrals between atomic electronic densities for the several definitions of AIM, for a number of molecular species. Do the same for the square roots of the densities.

(3) Atomic characteristics for various AIM recipes: Systematically and accurately explore various atomic properties in the several extant AIM models, especially those of Bader and Hirshfeld. Give special emphasis to transferability questions. 
(4) The right ensemble for AIM: An AIM is an open system (as opposed to closed), imbedded in an environment that is a bath of sorts, and such an atom can bear a non-integer number of electrons. Strive to determine the best way to describe the environment of an AIM in such a case, and how to define the bath as ones in the thermodynamic or statistical mechanical sense.

(5) $T_{c}$ : Systematically and accurately calculate and examine values of $T_{c}=T-T_{s}$ for a large number of atomic and molecular systems, and find empirical and/or theoretical formulas for predicting $T_{c}$.

(6) Local temperature: Local temperature at a point in a molecule has been variously defined in terms of local kinetic energy (a quantity itself variously defined). Try to pin this concept down, and elucidate its utility.

(7) Entropy and information: The density functional $T, T_{s}$, entropy, information and local temperature, are related concepts. Develop and refine and test this group of ideas and as appropriate incorporate them into the general DFT framework.

(8) Significance of the quantity $(\rho v / r)$ : Here $v$ is the external potential due to nuclei. For atoms, addition of a first-order term of this form is known to improve the gradient expansion of the kinetic energy, and a similar term improves the gradient expansion of the exchange energy. Explore its applications to molecules, and try to single it out of exact DFT theory for heterogeous systems. Or, try putting $v$ itself into future functionals.

(9) Density functional prediction of equilibrium bond lengths: There should be a simple model way to predict bond lengths from the properties of the atoms being bound, without using wavefunctions. Find this way. Of course, bond lengths can be calculated, but that is not the solution of this problem.

(10) Geometric structure and Kohn-Sham orbitals from diffraction experiments: We now know how to determine all Kohn-Sham properties from an exact density, including the potential due to nuclei. So, develop doing the same from experimental X-ray diffraction data.

(11) Shape-function DFT: For coulomb systems, the shape function, density divided by electron number $N$, determines everything (including $N$ ). This presents a whole theory that merits going into. Do it, though you will find company.

(12) Coulomb-system DFT: There may well exist asyet-undiscovered helpful consequences for systems for which the external potential is coulombic. Find some.
(13) Electron density to total energy without approximation: For any reasonable ground-state density or an approximation to it, knowing as we do how to go to accurate Kohn-Sham orbitals, effective potential, orbital energies, and kinetic energy, we would like to proceed further to get without approximation the correct total energy. Find how to do this. (Please let me be the first one to hear how, because I have been struggling with this problem, largely unsuccessfully for about a decade.)

(14) Model chemistries for the 21st Century: In the 20th Century, John Pople demonstrated the power of a model-system strategy: Carefully collect and stick with a large set of reference experimental data. Adopt as ultimate objective the accurate fitting of the advancing theory to these data. Take as good a start as possible, stating precisely what theoretical method you are using, and compute everything in the data set. Evaluate the results. Relax, then repeat with a new wrinkle improving the theoretical method (the "model"), and with a more sophisticated computer if possible, everything precisely defined. Repeat, repeat again. Again, and let the years roll by. As my last problem, I call on the theoretical chemistry community to carry forward Pople's strategy, even as chemical processes of all kinds are modeled better and better. And, as Robert Mulliken once said, be patient.

\section{Discussion}

Bryce Crawford, my $\mathrm{Ph} \mathrm{D}$ advisor and a famously effective graduate dean, once mentioned to me that he thought that a good $\mathrm{Ph} \mathrm{D}$ problem should have no more than something like $90 \%$ chance of success. This does not suggest, however, that $10 \%$ of $\mathrm{Ph} \mathrm{D}$ students should end ABT (all but thesis), because a good advisor of course rescues an ABT student by coming up with a revised project. I do not guarantee that one (or more!) of the problems above is not an ABT trap. The reader will have noticed that these problem statements do not provide recipes for proceeding, but rather simple suggestions as to what research might be undertaken in particular directions. One never knows to where a study will lead.

Computation will be involved in most of these problems, but their goals rarely have much directly to do with computation. The overall intent is to increase computing power, but it also is to round out the conceptual side. For example, should it work out convincingly, one hopes to bring entropy onto the scene. Also, continuing exposition of variational princi- 
ples is of the utmost importance. (Recall the classical thermodynamic principle that the Gibbs free energy of a homogeneous system, constrained to stay at constant temperature and pressure, will tend to a minimum - what more important principle is there in all chemical thermodynamics? In DFT the energy of an isolated molecule tends to minimize, yes, but what is the principle for a molecule held at constant chemical potential?) Apt theoretical physics and correct mathematical physics must be invoked in the further formal development of density-functional theory.
Many published papers deal in some way or other with these individual problems. Specific references are omitted in the listings above, because my knowledge is incomplete and important contributions would be missing. One also could argue that studying literature provides a budding scientist an excellent way to start research. My apologies to everyone who should have been referenced, and my heartfelt thanks to the hundreds of these people with whom I have discussed density-functional theory over the years. 